\title{
Spin-Dependent Transport in Carbon Nanotubes with Chromium Atoms
}

\author{
Stanislav P. Repetsky ${ }^{1}$, Oleg V. Tretyak ${ }^{2}$, Iryna G. Vyshivanaya², Dmitriy K. Cheshkovskiy ${ }^{1}$ \\ ${ }^{1}$ Department of Physics, Taras Shevchenko National University of Kyiv, Kyiv, Ukraine \\ ${ }^{2}$ Institute of High Technologies, Taras Shevchenko National University of Kyiv, Kyiv, Ukraine \\ Email: srepetsky@univ.kiev.ua
}

Received 27 August 2014; revised 25 September 2014; accepted 21 October 2014

Copyright @ 2014 by authors and Scientific Research Publishing Inc.

This work is licensed under the Creative Commons Attribution International License (CC BY).

http://creativecommons.org/licenses/by/4.0/

c) (†) Open Access

\begin{abstract}
Method is developed for self-consistent calculation of the energy spectrum of free energy and electrical disordered crystals. Processes of electron scattering on the ionic core potential of different sort, fluctuations of charge, spin density and lattice vibrations are taken into account. Electronic states of the system are described using tight binding multiband model. The nature of the spin-dependent electron transport of carbon nanotubes with chromium atoms adsorbed on the surface is explained. The value of the spin polarization of electron transport is determined by the difference of the partial densities of states of electrons with opposite spin projection at the Fermi level and the difference between the relaxation times of electron states. The value of the spin polarization of the electric current increases with increasing of $\mathrm{Cr}$ atoms concentration and magnitude of the external magnetic field.
\end{abstract}

\section{Keywords}

Spin-Dependent Transport, Carbon Nanotubes with Chromium Atoms, Tight Binding Model, Localized Magnetic Moment, Free Energy, Electrical Conductivity Tensor

\section{Introduction}

After a relatively recently discovered phenomenon of spin-dependent transport research of electrons spin polarization became actual [1]. Much attention has focused on systems based on carbon doped with transition metals. To clarify the mechanism of spin-dependent transport various theoretical models are intensively investigated. In particular, in [1] theoretically high spin polarization of electrons was predicted in carbon nanotubes, and [2] predicted theoretically almost $100 \%$ spin polarization of the electric current in carbon nanotubes with impurities of transition metals $\mathrm{Cr}$, V. One of the possible ways of changing properties of carbon nanotubes in order to apply them in nanoelectronics and spintronics is the application of admixtures of other elements. The presence of 
impurities can lead to a lowering of the symmetry of the crystal lattice and the degeneracy in the electron energy spectrum, as well as the appearance of additional energy gaps, whose width depends on the type of impurities and their concentrations. However, the effect of impurities on the electronic structure and related properties of carbon nanotubes are investigated insufficiently.

In our work, on the basis of a self-consistent tightbinding model electronic structure, free energy, conductivity and spin-dependent transport of carbon nanotubes with chromium impurity are investigated. Electron scattering on potentials of the ionic cores of different types, and the fluctuations of the spin of the electron density and lattice vibrations are taken into account.

In present literature, the studies of spin-dependent transport in carbon nanotubes are qualitatively described on the basis of ideas about the appearance of a gap in energy spectrum near Fermi level [2]. In our paper, numerical calculations of the spin polarization of electric current in carbon nanotubes are held on the basis of rigorous expressions for conductivity.

\section{Tensor of Electrical Conductivity}

The method of calculation of the energy spectrum of electrons and phonons, free energy and electrical conductivity of disordered crystal described in [3]-[5].

Electronic correlations in crystals are described in the multi-zone model of strong coupling. Considered the processes of electron scattering on the potential of ionic skeleton, fluctuations of the charge and spin density and lattice vibrations.

Using the formula Kubo, diagram technique for two-time temperature Green's function and neglecting the contribution of scattering processes on clusters of three or more sites for static electrical conductivity tensor we can get the expression [3]-[5]:

$$
\begin{aligned}
& \sigma_{\alpha \beta}=\frac{\mathrm{e}^{2} \hbar}{4 \pi V_{1}}\left\{\int _ { - \infty } ^ { \infty } \mathrm { d } \varepsilon _ { 1 } \frac { \partial f } { \partial \varepsilon _ { 1 } } \sum _ { s , s ^ { \prime } = + , - } ( 2 \delta _ { s s ^ { \prime } } - 1 ) \sum _ { \sigma \gamma , i } \left\{\left[v_{\beta} \tilde{K}\left(\varepsilon_{1}^{s}, v_{\alpha}, \varepsilon_{1}^{s^{\prime}}\right)\right]\right.\right. \\
& +\sum_{\lambda, m_{\lambda i}} P_{0 i}^{\lambda m_{\lambda i}} \tilde{K}\left(\varepsilon_{1}^{s^{\prime}}, v_{\beta}, \varepsilon_{1}^{s}\right)\left(t_{0 i}^{\lambda m_{\lambda i}}\left(\varepsilon_{1}^{s}\right) \tilde{K}\left(\varepsilon_{1}^{s}, v_{\alpha}, \varepsilon_{1}^{s^{\prime}}\right) t_{0 i}^{\lambda m_{\lambda i}}\left(\varepsilon_{1}^{s^{\prime}}\right)\right. \\
& +\sum_{\lambda, m_{\lambda i}} P_{0 i}^{\lambda m_{\lambda i}} \sum_{\substack{l j \neq 0 i, \lambda \lambda^{\prime}, m_{\lambda^{\prime} j}}} P_{l j 0 i}^{\lambda^{\prime} m_{\lambda^{\prime} j} / \lambda m_{\lambda i}}\left[\left[\tilde{K}\left(\varepsilon_{1}^{s^{\prime}}, v_{\beta}, \varepsilon_{1}^{s}\right) v_{\alpha} \tilde{G}\left(\varepsilon_{1}^{s^{\prime}}\right)\right] \times T^{(2) \lambda m_{\lambda i} 0 i, \lambda^{\prime} m_{\lambda^{\prime} j} j j}\left(\varepsilon_{1}^{s^{\prime}}\right)\right. \\
& +\left[\tilde{K}\left(\varepsilon_{1}^{s^{\prime}}, v_{\beta}, \varepsilon_{1}^{s}\right) v_{\alpha} \tilde{G}\left(\varepsilon_{1}^{s^{\prime}}\right)\right] T^{(2) \lambda^{\prime} m_{\lambda^{\prime} j} l j, \lambda m_{\lambda i} 0 i}\left(\varepsilon_{1}^{s^{\prime}}\right)+\left[\tilde{K}\left(\varepsilon_{1}^{s}, v_{\alpha}, \varepsilon_{1}^{s^{\prime}}\right) v_{\beta} \tilde{G}\left(\varepsilon_{1}^{s}\right)\right] T^{(2) \lambda m_{\lambda i} 00 i, \lambda^{\prime} m_{\lambda^{\prime} j} j j}\left(\varepsilon_{1}^{s}\right) \\
& +\left[\tilde{K}\left(\varepsilon_{1}^{s}, v_{\alpha}, \varepsilon_{1}^{s^{\prime}}\right) v_{\beta} \tilde{G}\left(\varepsilon_{1}^{s}\right)\right] T^{(2) \lambda^{\prime} m_{\lambda^{\prime} j} l j, \lambda m_{\lambda i} 0 i}\left(\varepsilon_{1}^{s}\right)+\tilde{K}\left(\varepsilon_{1}^{s^{\prime}}, v_{\beta}, \varepsilon_{1}^{s}\right)\left[\left(t_{l j}^{\lambda^{\prime} m_{\lambda^{\prime} j}}\left(\varepsilon_{1}^{s}\right) \tilde{K}\left(\varepsilon_{1}^{s}, v_{\alpha}, \varepsilon_{1}^{s^{s^{\prime}}}\right) t_{0 i}^{\lambda m_{\lambda i}}\left(\varepsilon_{1}^{s^{\prime}}\right)\right.\right. \\
& +t_{l j}^{\lambda^{\prime} m_{\lambda^{\prime} j}}\left(\varepsilon_{1}^{s}\right) \tilde{K}\left(\varepsilon_{1}^{s}, v_{\alpha}, \varepsilon_{1}^{s^{\prime}}\right) T^{(2) \lambda m_{\lambda i} 0 i, \lambda^{\prime} m_{\lambda^{\prime} j} l j}\left(\varepsilon_{1}^{s^{\prime}}\right)+T^{(2) \lambda^{\prime} m_{\lambda^{\prime} j} l j, \lambda m_{\lambda i} 0 i}\left(\varepsilon_{1}^{s}\right) \tilde{K}\left(\varepsilon_{1}^{s}, v_{\alpha}, \varepsilon_{1}^{s^{\prime}}\right) t_{0 i}^{\lambda m_{\lambda i}}\left(\varepsilon_{1}^{s^{\prime}}\right) \\
& +T^{(2) \lambda^{\prime} m_{\lambda^{\prime} j} l j, \lambda m_{\lambda i} 0 i}\left(\varepsilon_{1}^{s}\right) \tilde{K}\left(\varepsilon_{1}^{s}, v_{\alpha}, \varepsilon_{1}^{s^{\prime}}\right) T^{(2) \lambda m_{\lambda i} 0 i, \lambda^{\prime} m_{\lambda^{\prime} j} l j}\left(\varepsilon_{1}^{s^{\prime}}\right) \\
& \left.\left.\left.\left.+T^{(2) \lambda^{\prime} m_{\lambda^{\prime} j} l j, \lambda m_{\lambda i} 0 i}\left(\varepsilon_{1}^{s}\right) \tilde{K}\left(\varepsilon_{1}^{s}, v_{\alpha}, \varepsilon_{1}^{s^{\prime}}\right) T^{(2) \lambda^{\prime} m_{\lambda^{\prime} j} l j, \lambda m_{\lambda i} 0 i}\left(\varepsilon_{1}^{s^{\prime}}\right)\right)\right]\right]\right\}^{0 i \gamma \sigma, 0 i \gamma \sigma} \\
& \left.+\int_{-\infty}^{\infty} \int_{-\infty}^{\infty} \mathrm{d} \varepsilon_{1} \mathrm{~d} \varepsilon_{2} f\left(\varepsilon_{1}\right) f\left(\varepsilon_{2}\right)\left\langle\Delta G_{\alpha \beta}^{I I}\left(\varepsilon_{1}, \varepsilon_{2}\right)\right\rangle\right\},
\end{aligned}
$$

where

$$
\begin{gathered}
\tilde{K}\left(\varepsilon_{1}^{s}, v_{\alpha}, \varepsilon_{1}^{s^{\prime}}\right)=\tilde{G}\left(\varepsilon_{1}^{s}\right) v_{\alpha} \tilde{G}\left(\varepsilon_{1}^{s^{\prime}}\right), \\
\tilde{G}\left(\varepsilon_{1}^{+}\right)=\tilde{G}_{r}\left(\varepsilon_{1}\right) \\
\tilde{G}\left(\varepsilon_{1}^{-}\right)=\tilde{G}_{a}\left(\varepsilon_{1}\right)=\left(\tilde{G}_{r}\right)^{*}\left(\varepsilon_{1}\right),
\end{gathered}
$$

$\tilde{G}_{r}(\varepsilon), \tilde{G}_{a}(\varepsilon)$ is retarded and advanced one-particle Green functions of effective medium respectively.

The first component in the right-hand side of expression (1) describes the contribution of electroconductivity 
approximation in the coherent potential. Other components describes the contribution in electroconductivity processes of electrons scattering on clusters consisting two atoms.

In the formula (1) component $\Delta G_{\alpha \beta}^{I I}\left(\varepsilon_{1}, \varepsilon_{2}\right)$ of two-particle Green's function is caused by the electron-electron interaction and has the form:

$$
\begin{aligned}
& \Delta G_{\alpha \beta}^{I I}\left(\varepsilon_{1}, \varepsilon_{2}\right)= \\
& \frac{i}{2 \pi} v_{\alpha n_{4} n_{2}} v_{\beta n_{3} n_{1}}\left\{\left[G_{r n_{1} n_{6}}\left(\varepsilon_{1}\right)-G_{a n_{1} n_{6}}\left(\varepsilon_{1}\right)\right]\left[G_{r n_{2} n_{5}}\left(\varepsilon_{2}\right)-G_{a n_{2} n_{5}}\left(\varepsilon_{2}\right)\right]\left[G_{a n_{7} n_{4}}\left(\varepsilon_{2}\right) G_{r n_{8} n_{3}}\left(\varepsilon_{1}\right)-G_{r n n_{7}}\left(\varepsilon_{2}\right) G_{a n_{8} n_{3}}\left(\varepsilon_{1}\right)\right]\right. \\
& \quad+G_{a n_{1} n_{6}}\left(\varepsilon_{1}\right)\left[G_{r n_{2} n_{5}}\left(\varepsilon_{2}\right)-G_{a n_{2} n_{5}}\left(\varepsilon_{2}\right)\right] G_{a n_{7} n_{4}}\left(\varepsilon_{2}\right)\left[G_{r n_{8} n_{3}}\left(\varepsilon_{1}\right)-G_{a n_{8} n_{3}}\left(\varepsilon_{1}\right)\right] \\
& \quad-G_{r n_{1} n_{6}}\left(\varepsilon_{1}\right)\left[G_{r n_{2} n_{5}}\left(\varepsilon_{2}\right)-G_{a n_{2} n_{5}}\left(\varepsilon_{2}\right)\right] G_{r n_{7} n_{4}}\left(\varepsilon_{2}\right)\left[G_{r n_{8} n_{3}}\left(\varepsilon_{1}\right)-G_{a n_{8} n_{3}}\left(\varepsilon_{1}\right)\right] \\
& \quad+\left[G_{a n_{1} n_{6}}\left(\varepsilon_{1}\right) G_{r n_{2} n_{5}}\left(\varepsilon_{2}\right)-G_{r n_{1} n_{6}}\left(\varepsilon_{1}\right) G_{a n_{2} n_{5}}\left(\varepsilon_{2}\right)\right]\left[G_{r n n_{4}}\left(\varepsilon_{2}\right)-G_{a n n_{4} n_{4}}\left(\varepsilon_{2}\right)\right]\left[G_{r n_{8} n_{3}}\left(\varepsilon_{1}\right)-G_{a n_{8} n_{3}}\left(\varepsilon_{1}\right)\right] \\
& \quad+\left[G_{r n_{1} n_{6}}\left(\varepsilon_{1}\right)-G_{a n_{1} n_{6}}\left(\varepsilon_{1}\right)\right] G_{r n_{2} n_{5}}\left(\varepsilon_{2}\right)\left[G_{r n_{7} n_{4}}\left(\varepsilon_{2}\right)-G_{a n_{7} n_{4}}\left(\varepsilon_{2}\right)\right] G_{r n_{8} n_{3}}\left(\varepsilon_{1}\right) \\
& \left.\quad-\left[G_{r n_{1} n_{6}}\left(\varepsilon_{1}\right)-G_{a n_{1} n_{6}}\left(\varepsilon_{1}\right)\right] G_{a n_{2} n_{5}}\left(\varepsilon_{2}\right)\left[G_{r n_{7} n_{4}}\left(\varepsilon_{2}\right)-G_{a n_{7} n_{4}}\left(\varepsilon_{2}\right)\right] G_{a n_{8} n_{3}}\left(\varepsilon_{1}\right)\right\} \Gamma_{n_{5} n_{8}}^{n_{n} n_{7}}\left(\varepsilon_{1}, \varepsilon_{2} ; \varepsilon_{2}, \varepsilon_{1}\right) .
\end{aligned}
$$

where $\Gamma_{n_{5} n_{8}}^{n_{6} n_{7}}\left(\varepsilon_{1}, \varepsilon_{2} ; \varepsilon_{2}, \varepsilon_{1}\right)$ is vertex part of diagram for the mass operator of electron-electron interaction, $(n \equiv n i \gamma \sigma)$, here $n$ is number of primitive cell, $i$-the sublattice-site number in primitive cell, $\gamma$-energy band index, $\sigma=1 / 2,-1 / 2$ - quantum number, which defines value of spin projection on $z$ axis.

Operator $\alpha$-the projection of the electron velocity in (1) is:

$$
v_{\alpha}(\boldsymbol{k})=\frac{1}{\hbar} \frac{\partial H_{0}^{(1)}(\boldsymbol{k})}{\partial k_{\alpha}} .
$$

To simplify the formula (1) we use approximate expression for averaged value $\left\langle\Delta G_{\alpha \beta}^{I I}\left(\varepsilon_{1} ; \varepsilon_{2}\right)\right\rangle \approx \Delta \tilde{G}_{\alpha \beta}^{I I}\left(\varepsilon_{1} ; \varepsilon_{2}\right)$, where $\Delta \tilde{G}_{\alpha \beta}^{I I}\left(\varepsilon_{1} ; \varepsilon_{2}\right)$-is derived from the expression (2) by replacing the Green function $G(\varepsilon)$ with Green function of effective medium $\tilde{G}(\varepsilon)$.

In formula (1):

$$
\begin{aligned}
& t^{\lambda, m_{\lambda_{i}} 0 i}=\left[I-\left(\Sigma^{\lambda, m_{\lambda_{i}} 0 i}-\sigma^{0 i}\right) \tilde{G}\right]^{-1}\left(\Sigma^{\lambda, m_{\lambda_{i}} 0 i}-\sigma^{0 i}\right), \\
& T^{(2) \lambda, m_{\lambda, i} 0 i, \lambda^{\prime} m_{\lambda^{\prime} j} l j}=\left[I-t^{\lambda, m_{\lambda, i} 0 i} \tilde{G} t^{\lambda^{\prime} m_{\lambda^{\prime} j} l j} \tilde{G}\right] t^{\lambda, m_{\lambda, i} 0 i} \tilde{G} t^{\lambda^{\prime} m_{\lambda^{\prime} j} l j}\left[I+\tilde{G} t^{\lambda, m_{\lambda, i} 0 i}\right] .
\end{aligned}
$$

where

$t^{\lambda m_{\lambda i} 0 i}$ is scattering operator on the one site,

$T^{(2) \lambda m_{\lambda i} i i, \lambda^{\prime} m_{\lambda^{\prime} j} j^{j j}}$ is scattering operator on the two sites,

$\sigma^{0 i}$ is coherent potential that describes effective medium,

$\lambda, m_{\lambda_{i}}$ is atom sort and magnetic moment projection on $z$ axis respectively.

The value $\Sigma_{e}^{n_{i_{1}}}(\varepsilon)$ in the expression for the single-center scattering operator $t^{n_{1} i_{1}}$ that describes the scattering on potentials of ionic skeleton, static charge fluctuations and spin density is given by formula:

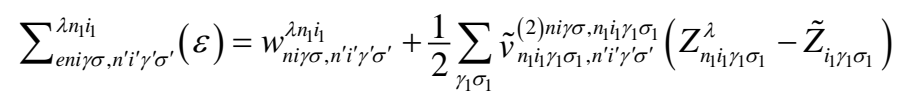

where $Z_{n_{1} \gamma_{1} \sigma_{1}}^{\lambda}, \tilde{Z}_{i_{1} \gamma_{1} \sigma_{1}}$ is number of electrons in $n_{1} i_{1} \gamma_{1} \sigma_{1}$ state for $\lambda$ atom in crystal and effective medium respectively, $\quad \tilde{v}_{n_{1} i_{1} / \sigma_{1}, n^{\prime} i^{\prime} \gamma^{\prime} \sigma^{\prime}}^{(n)}$ is electron-electron interaction operator's matrix element [3]-[5].

In expression (1) values $P_{j l i 0}^{\lambda^{\prime} \lambda}=P_{i 0}^{\lambda} P_{j l i 0}^{\lambda^{\prime} / \lambda}, \quad P_{j l i 0}^{m_{\lambda^{\prime}} m_{\lambda i}}=P_{i 0}^{m_{\lambda i}} P_{j l i 0}^{m_{\lambda^{\prime} j} / m_{\lambda i}}$-the probability of finding atoms of different sorts and values of localized magnetic moment projection at the lattice sites [3]-[5]. In the Hamiltonian of the system should be included term that is the operator of interaction energy of intrinsic magnetic moment of a particle with an external magnetic field.

Spin-dependent transport in systems with strong electronic correlations is described by partial constituent 
of diagonal conductivity tensor element (1), which corresponds to a specific value of the electron spin projection.

\section{Results of Calculations and Conclusions}

Here are the results of calculation of the energy spectrum of electrons and phonons and conductivity of carbon nanotubes doped with chromium. In calculation, renormalization of vertex parts of mass operator of electron-electron and electron-phonon interactions [3]-[5] was neglected. Real wave functions of $2 \mathrm{~s}, 2 \mathrm{p}, 3 \mathrm{~s}$, 3d-states of neutral non-interacting atoms of carbon were chosen. The off-diagonal matrix elements $(n i)$ by site index of Hamiltonian calculated by taking into account the first three coordination spheres. Contribution to the static displacements of atoms and members was neglected in the calculations. Calculations were performed for the temperature $T=300 \mathrm{~K}$.

We performed geometry optimization of the crystal structure of carbon nanotube of chirality $(3,0)$ with $\mathrm{Cr}$ impurity. Geometric optimization of the crystal structure was achieved by minimizing the free energy. Carbon nanotube doped with $\mathrm{Cr}$ has a one-dimensional crystal structure. Primitive cell contains 18 non-equivalent atom positions. Carbon atoms are located in 12 positions on the surface of the inner cylinder. The distance between the carbon atoms is $0.142 \mathrm{~nm}$. Cr atoms are randomly located in the 6 position on the outer surface of the cylinder opposite the center of a hexagon, the vertices of which are carbon atoms. Through the study of free energy minimum found that $\mathrm{Cr}$ atoms are randomly located on the surface of nanotubes. This indicates that the probability of $\mathrm{Cr}$ atoms arrangement $P_{l j 0 i}^{\lambda^{\prime} \lambda}=P_{l j}^{\lambda^{\prime}}=c^{\lambda^{\prime}}$, where $c^{\lambda^{\prime}}$ - the ratio of $\mathrm{Cr}$ atoms with respect to the six possible positions of the atoms $\mathrm{Cr}$ within the primitive cell. The distance between carbon atoms and $\mathrm{Cr}$ is 0.22 $\mathrm{nm}$. The relative position of carbon atoms and $\mathrm{Cr}$ is similar to the location of atoms of transition metals on the surface of carbon nanotubes of large diameter, which are described in [1] [2] by ultrasoft pseudopotential method [6] [7].

The value localized magnetic moment projection of the atom $\mathrm{Cr}$ and induced localized magnetic moment of an atom $\mathrm{C}$ in the direction of the magnetic field increases with the size of the field. For carbon nanotubes of $5 \mathrm{Cr}$ atoms in primitive cell value projection magnetic moment of the atom $\mathrm{Cr}$ varies within $m_{\mathrm{Cr}}=(1.02 ; 2,24) \mu_{B}$, and the magnetic moment of the atom $\mathrm{C}$-within $m_{C}=(0,0036 ; 0,02) \mu_{B}$ with increasing values of the magnetic field from zero to $H=200 \mathrm{~A} / \mathrm{m}$. The magnetic field is oriented along the axis of the carbon nanotube. Parameter of pair correlations in the orientation of localized magnetic moments on lattice sites for the first coordination sphere in the absence of magnetic field equals to $\varepsilon^{m}=0.235$. The value $\varepsilon^{m}$ for the second and third coordination spheres is close to zero. A positive value of $\varepsilon^{m}$ for the first coordination sphere indicates that the localized magnetic moment given carbon atom is oriented in the same direction as the magnetic moment of the nearest $\mathrm{Cr}$ atom.

Figure 1 shows a partial $g_{e \sigma}(\varepsilon)=\frac{1}{v} \sum_{i, \gamma, \lambda} P_{0 i}^{\lambda} g_{0 i \gamma \sigma}^{\lambda}(\varepsilon)$ and full $g_{e}(\varepsilon)=\sum_{\sigma} g_{e \sigma}(\varepsilon)$ densities of electron states of carbon nanotube with an admixture of $\mathrm{Cr}$ in the absence of external magnetic field. In the absence of a magnetic field $g_{1 / 2}(\varepsilon)=g_{-1 / 2}(\varepsilon)$. Vertical line shows the Fermi level $\varepsilon_{F}$.

In Figure 2, points show the dependence of the free energy $F$ on the parameter of pair correlations in the arrangement of $\mathrm{Cr}$ impurities on lattice sites $\varepsilon^{B B}=\varepsilon_{l j 0 i}^{B B}$ for the first coordination sphere. Atom of $\mathrm{Cr}$ is denoted as atom of sort $B$. The dependence $F\left(\varepsilon^{B B}\right)$ is shown in the region of free energy minimum. The free energy is measured from electrostatic interaction energy of ions of sort A.

Figure 3 shows partial $g_{e \sigma}(\varepsilon)$ and full $g_{e}(\varepsilon)$ densities of electron states of carbon nanotube with 5 atoms of Cr per primitive cell in external magnetic field $H=100 \mathrm{~A} / \mathrm{m}$.

Figure 3 shows part of the energy spectrum that is close to the Fermi level.

As shown in Figure 3, for a given sign of the projection of localized magnetic moment on sites of lattice energies of the electron with spin $\sigma=1 / 2$ shifted relative to values of the energy of an electron with spin $\sigma=-1 / 2$. This leads to the formation of the Coulomb gap in the energy spectrum of electrons, which is visible on $g(\varepsilon)$ of Figure 3. The results presented in Figure 3, qualitatively consistent with results obtained by another method in [2].

In Figure 4, the dependence of the spin polarization electric current $\Delta \sigma / \sigma=\left(\sigma_{1 / 2}-\sigma_{-1 / 2}\right) / \sigma$ of carbon nanotube with chirality $(3,0)$ and 5 atoms of $\mathrm{Cr}$ per primitive cell on the magnitude of the external magnetic field calculated by the formula (1) for temperature $300 \mathrm{~K}$ is shown. 


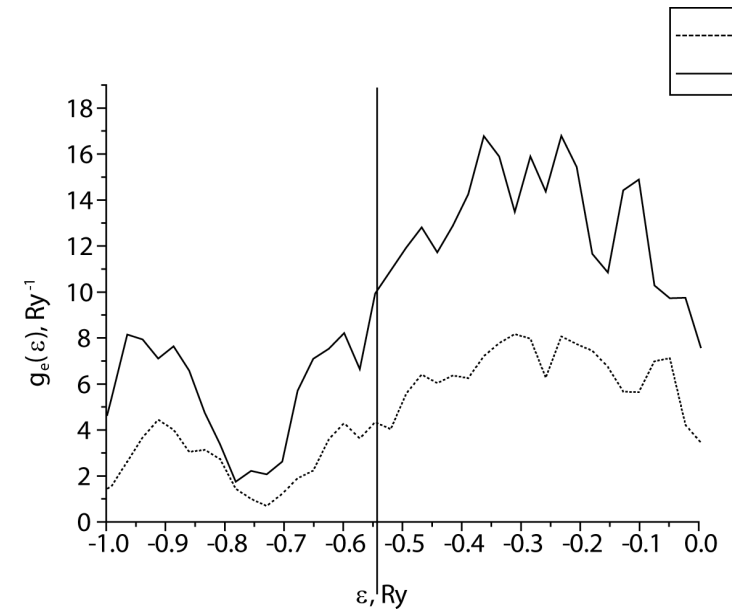

Figure 1. Densities of electron states of carbon nanotube with an admixture of Cr.

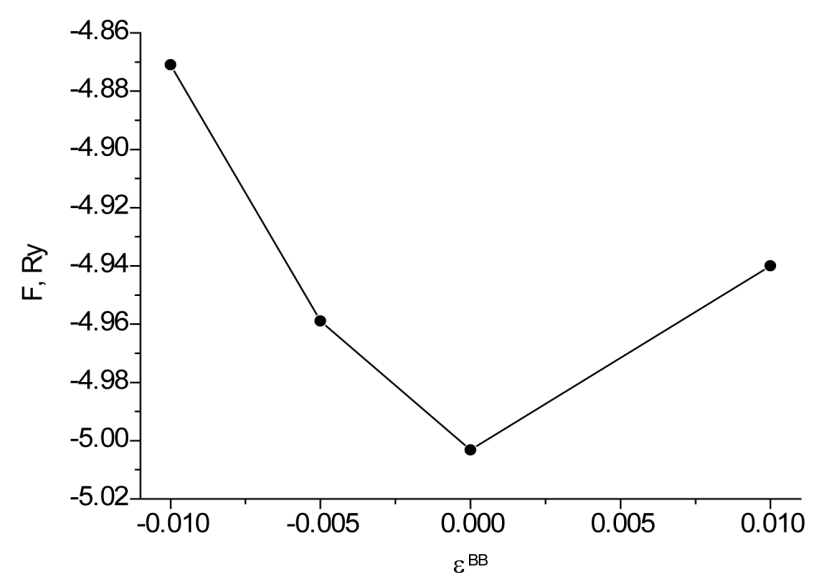

Figure 2. Dependence of free energy $F$ for carbon nanotubes with 5 atoms of $\mathrm{Cr}$ per primitive cell on parameter of pair correlations in the arrangement of $\mathrm{Cr}$ impurities on lattice sites $\varepsilon^{B B}$.

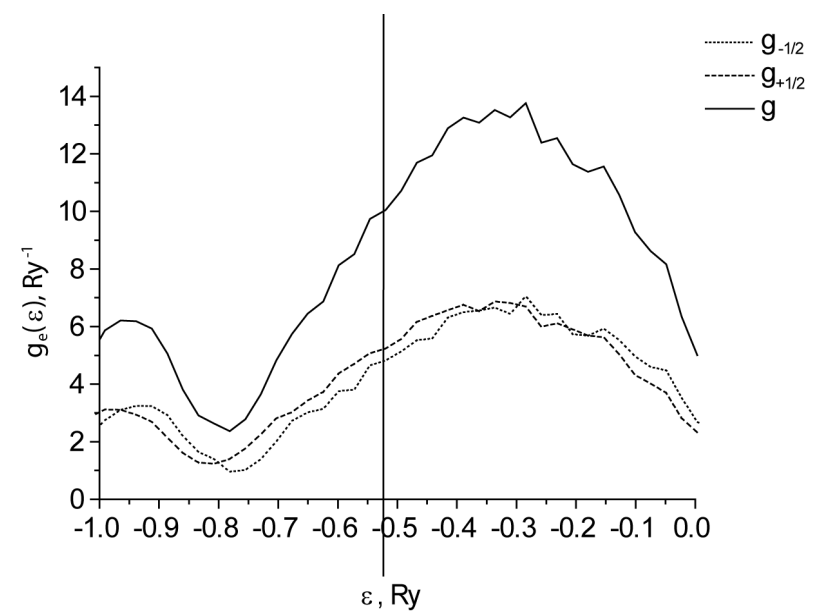

Figure 3. Densities of electron states of carbon nanotube with 5 atoms of $\mathrm{Cr}$ per primitive cell in external magnetic field $H=100 \mathrm{~A} / \mathrm{m}$. 


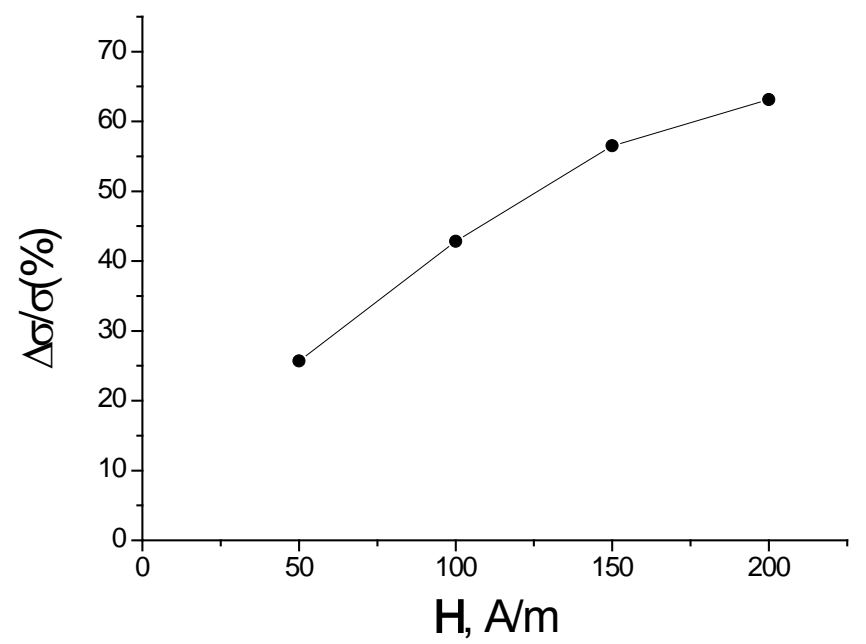

Figure 4. The dependence of spin polarized electric current $\Delta \sigma / \sigma$ of carbon nanotube on the magnitude of the external magnetic field $H$.

Thus, the phenomenon of spin-dependent electron transport in a carbon nanotube is the result of strong electron correlations caused by the presence of chromium atoms. The value of the spin polarization of electron transport determined by the difference of the partial densities of states (Figure 3) electrons with opposite spin projection at the Fermi level and the difference between the relaxation times arising from different occupation numbers of single-electron states $Z_{n_{1} \gamma_{1} \gamma_{1} \sigma_{1}}^{\lambda}$ of atoms of carbon and chromium. The value of the spin polarization of the electric current increases with increasing of $\mathrm{Cr}$ atoms concentration and magnitude of the external magnetic field. In [1] calculated the electronic structure and properties of carbon nanotubes with transition metal chains, adsorbed on the surface, is based on density functional method using ultra-soft pseudopotential. Our results are qualitatively consistent with the results of [1], in which $a b$-initio electron density functional method shows that the chains of transition metals adsorbed on the surface of carbon nanotubes, open a gap in the electrons states with a certain spin value.

\section{References}

[1] Durgun, E. and Ciraci, S. (2006) Physical Review B, 74, Article ID: 125404. http://dx.doi.org/10.1103/PhysRevB.74.125404

[2] Yang, C., Zhao, J. and Lu, J.P. (2004) Nano Letters, 4, 561-563. http://dx.doi.org/10.1021/nl035104x

[3] Repetsky, S.P. and Shatnii, T.D. (2002) Theoretical and Mathematical Physics, 131, 832.

[4] Repetskii, S.P. and Vyshivanaya, I.G. (2005) Physics of Metals and Metallography, 99, 558.

[5] Repetskii, S.P., Vyshivanaya, I.G. and Cheshkovskii, D.K. (2012) Physics of Metals and Metallography, 113, $213-221$. http://dx.doi.org/10.1134/S0031918X12030143

[6] Vanderbilt, D. (1990) Physical Review B, 41, 7892. http://dx.doi.org/10.1103/PhysRevB.41.7892

[7] Laasonen, K., Car, R., Lee, C. and Vanderbilt, D. (1991) Physical Review B, 43, 6796. http://dx.doi.org/10.1103/PhysRevB.43.6796 
Scientific Research Publishing (SCIRP) is one of the largest Open Access journal publishers. It is currently publishing more than 200 open access, online, peer-reviewed journals covering a wide range of academic disciplines. SCIRP serves the worldwide academic communities and contributes to the progress and application of science with its publication.

Other selected journals from SCIRP are listed as below. Submit your manuscript to us via either submit@scirp.org or Online Submission Portal.
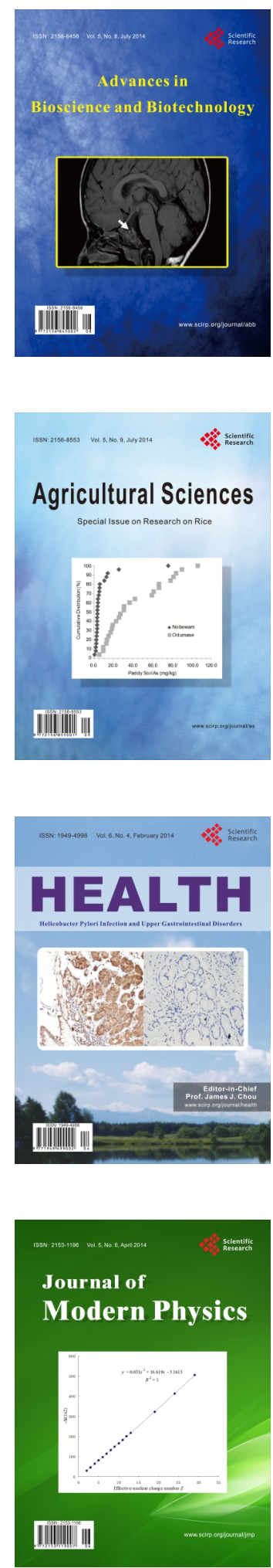
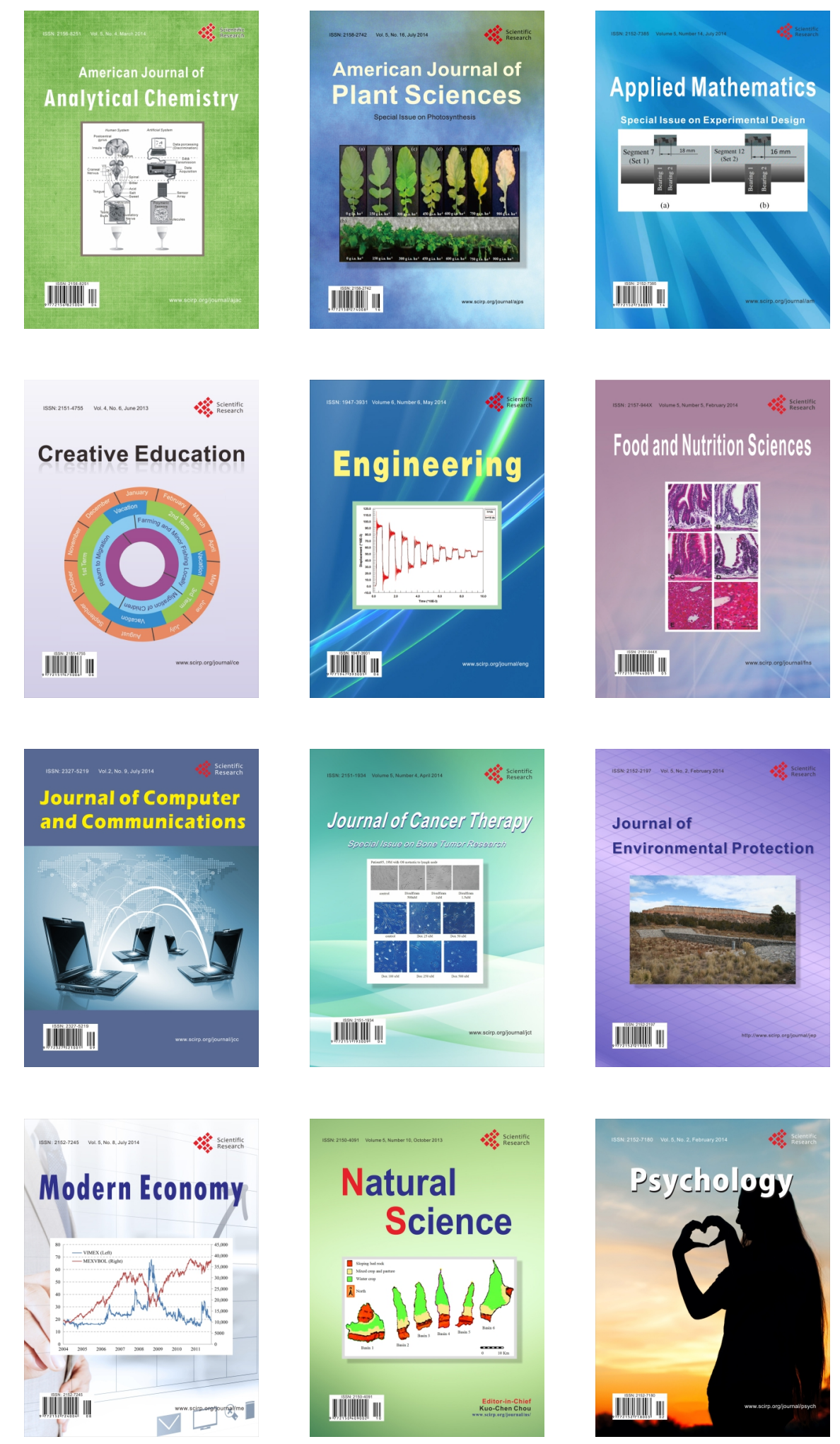